\title{
The effect of intermittent $v$. chronic energy restriction on weight loss and markers of chronic disease risk in premenopausal women: a randomised pilot trial
}

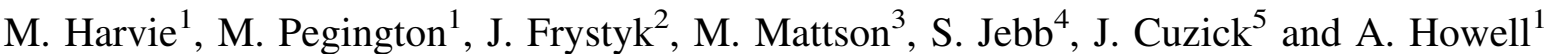 \\ ${ }^{1}$ Genesis Prevention Centre, University Hospital of South Manchester, Manchester M23 9LT, UK, ${ }^{2}$ Medical Research \\ Laboratories, Aarhus University, Denmark, ${ }^{3}$ Laboratory of Neurosciences, National Institute on Aging Intramural Research \\ Program, Baltimore, MD, USA, ${ }^{4}$ MRC Human Nutrition Research Group, Cambridge CB1 9NL, UK and \\ ${ }^{5}$ CRUK Department of Epidemiology and Statistics, Wolfson Institute, London EC1M 6B, UK
}

Excess adiposity increases risk of diseases such as diabetes, CVD, dementia and certain forms of cancer including breast cancer. Weight loss is likely to reduce risk of these diseases but is difficult to achieve and maintain. Animal studies indicate that intermittent energy restriction (IER) may be superior to continuous energy restriction (CER) for improving insulin sensitivity ${ }^{(1)}$ and reducing cancer risk ${ }^{(2)}$. It is hypothesised that IER may be superior to CER for reducing weight and improving insulin sensitivity, and may have beneficial effects on other biomarkers of disease risk, e.g. lipids, adipokines and inflammatory and oxidative stress markers and may also be more acceptable to women.

The effectiveness and acceptability of IER ( $n$ 53) $v$. CER ( $n$ 54) were tested in overweight or obese premenopausal women (mean age 40.1 (SD 3.9) years, mean adult weight gain 20.7 (SD 11.2) kg). Both diets provided $75 \%$ estimated energy requirements; CER subjects had approximately (approx) $6270 \mathrm{~kJ}(1500 \mathrm{kcal}) / \mathrm{d}$ for $7 \mathrm{~d} /$ week, IER subjects had approx $2665 \mathrm{~kJ}(550 \mathrm{kcal}) / \mathrm{d}$ for $2 \mathrm{~d} /$ week and approx $7524 \mathrm{~kJ}$ $(1800 \mathrm{kcal})$ for $5 \mathrm{~d} /$ week.

Eighteen women (17\%) withdrew from the study before 6 months (IER 11, CER 7; main reasons: stress 4, pregnancy 3, change in employment 3, couldn't stick to diet 3). Change in weight, body composition, anthropometry and biomarkers at 6 months for last observation carried forward are reported.

\begin{tabular}{|c|c|c|c|c|c|c|c|c|c|}
\hline \multirow[b]{3}{*}{ Variable } & \multicolumn{4}{|c|}{ IER } & \multicolumn{4}{|c|}{ CER } & \multirow[b]{3}{*}{$P$} \\
\hline & \multicolumn{2}{|c|}{ Baseline } & \multicolumn{2}{|c|}{6 Months } & \multicolumn{2}{|c|}{ Baseline } & \multicolumn{2}{|c|}{6 Months } & \\
\hline & Mean & SD & Mean & SD & Mean & SD & Mean & SD & \\
\hline Weight (kg) & 81.5 & 14.3 & 75.0 & 13.8 & 84.4 & 17.2 & 78.8 & 16.7 & 0.26 \\
\hline Body fat (kg) & 33.6 & 10.1 & 28.7 & 10.0 & 35.3 & 12.5 & 30.9 & 12.1 & 0.34 \\
\hline Fat-free mass (kg) & 47.6 & 5.0 & 46.2 & 4.6 & 49.1 & 5.2 & 47.9 & 5.1 & 0.21 \\
\hline Waist $(\mathrm{cm})$ & 102 & 13.3 & 95.1 & 13.0 & 103 & 13.8 & 97.4 & 13.9 & 0.13 \\
\hline TAG (mmol/l) & 1.2 & 0.7 & 1.0 & 0.4 & 1.3 & 0.7 & 1.0 & 0.5 & 0.60 \\
\hline HDL (mmol/l) & 1.5 & 0.3 & 1.5 & 0.3 & 1.6 & 0.4 & 1.5 & 0.4 & 0.24 \\
\hline $\mathrm{LDL}(\mathrm{mmol} / \mathrm{l})$ & 3.1 & 0.8 & 2.9 & 0.7 & 3.1 & 0.8 & 2.8 & 0.8 & 0.93 \\
\hline $\mathrm{CRP}(\mathrm{mg} / \mathrm{l}) \dagger$ & 4.5 & $3.8,5.4$ & 3.9 & $3.2,4.7$ & 3.7 & $3.2,4.3$ & 2.9 & $2.6,3.4$ & 0.15 \\
\hline $\operatorname{HOMA}(\mu \mathrm{U} / \mathrm{mmol} / \mathrm{l}) \dagger$ & 1.5 & $1.3,1.8$ & 1.1 & $0.9,1.3$ & 1.6 & $1.3,1.8$ & 1.3 & $1.1,1.6$ & $0.04 * *$ \\
\hline Adiponectin $(\mu \mathrm{g} / \mathrm{ml}) \dagger$ & 10.6 & $9.5,11.8$ & 11.8 & $10.4,13.3$ & 10.8 & $9.7,12.1$ & 10.9 & $9.7,12.3$ & 0.09 \\
\hline Leptin $(\mathrm{ng} / \mathrm{ml}) \dagger$ & 28.5 & $23.2,35.0$ & 16.0 & $12.6,20.4$ & 28.2 & $23.5,33.8$ & 17.1 & $13.5,21.7$ & 0.53 \\
\hline IGF-I (total; $\mu \mathrm{g} / \mathrm{l}) \dagger$ & 201 & 185,219 & 190 & 172,209 & 203 & 192,215 & 204 & 190,218 & 0.17 \\
\hline $\mathrm{BDNF}(\mathrm{pg} / \mathrm{ml})$ & 9539 & 2079 & 9264 & 1769 & 9898 & 1847 & 9528 & 1594 & 0.87 \\
\hline Ghrelin $(\mathrm{pg} / \mathrm{ml}) \dagger$ & 136 & 117,159 & 154 & 125,190 & 133 & 111,159 & 149 & 123,182 & 0.92 \\
\hline Ketones $(\mu \mathrm{M}) \dagger$ & 40.8 & $31.5,52.7$ & 66.4 & $49.0,90.0$ & 48.0 & $37.8,61.0$ & 51.2 & $39.4,66.7$ & 0.12 \\
\hline AOPP fast acting $(\mu \mathrm{M}) \dagger$ & 41.5 & $34.8,49.5$ & 35.0 & $30.4,40.3$ & 43.2 & $36.7,51.0$ & 37.0 & $31.7,43.2$ & 0.76 \\
\hline AOPP slow acting $(\mu \mathrm{M}) \dagger$ & 1.7 & $1.5,2.0$ & 1.6 & $1.3,1.8$ & 1.4 & $1.2,1.7$ & 1.7 & $1.5,1.9$ & 0.12 \\
\hline
\end{tabular}

CRP, C-reactive protein; HOMA, homeostasis model assessment of insulin resistance; IGF-I insulin-like growth factor; BDNF, brain-derived neurotrophic factor;

AOPP, advanced oxidation protein products. $* * P<0.05$. $\dagger$ Median and $95 \% \mathrm{CI}$.

IER gives similar results to CER in relation to weight loss and many of the risk variables measured and may be an alternative approach to CER. The superior reductions in insulin resistance with IER may be related to periods of very low energy intake (approx $70 \%$ restriction). Future studies will investigate the mechanism of IER and its optimal duration.

1. Anson RM, Guo Z, de Cabo R et al. (2003) Proc Natl Acad Sci U S A 100, 6216-6220.

2. Cleary MP, Jacobson MK, Phillips FC et al. (2002) Cancer Epidemiol Biomarkers Prev 11, 836-843. 